\title{
Nancy, Badiou y Lacan: la composición compleja del concepto
}

\author{
ROQUE FARRÁN
}

Universidad Nacional de Córdoba

Consejo Nacional de Investigaciones Científicas y Técnicas

roquefarran@gmail.com

\begin{abstract}
Resumen: En este artículo presentaremos primero dos discusiones muy puntuales que confrontan a Badiou con Nancy y Lacan en torno al vacío y al infinito. Mostraremos después cómo pensamos que se articula el concepto, tanto en filosofía como en psicoanálisis, a la luz de dichas complejidades y de las imbricadas relaciones entre los diversos dispositivos de pensamiento que ellas implican.
\end{abstract}

Palabras clave: vacío, infinito, composibilidad, psicoanálisis, nudo

\begin{abstract}
In this article we present first two arguments about the void and the infinity, confronting Badiou both with Nancy and Lacan. We propose then an explanation of how the concept is articulated, both in philosophy and in psychoanalysis, in the light of such complexities and imbricated relationships among the diverse thinking dispostives that they imply.
\end{abstract}

Key words: void, infinite, compossibility, psychoanalysis, knot

En este artículo partiremos de dos discusiones muy puntuales que confrontan, primero, a Badiou con Nancy en torno al vacío y, segundo, a Badiou con Lacan en torno al infinito. Después mostraremos cómo pensamos que se articula el concepto, tanto en filosofía como en psicoanálisis, a la luz de dichas complejidades y de las imbricadas relaciones entre los diversos dispositivos de pensamiento que ellas implican. Sin extendernos demasiado en preámbulos y presentaciones, quisiéramos introducir directamente los conceptos específicos de los autores mencionados, adaptados en función de las necesidades argumentativas del texto, para indicar los puntos problemáticos que en torno a ellos nos permitirán, después, efectuar nuestro propio aporte a su pensamiento. Por esta razón no apelaremos a datos biográficos, contextualizaciones más amplias o desarrollos generales acerca de la obra de los autores. Las discusiones teóricas serán puntuales y creemos que se pueden seguir con rigor más allá del conocimiento exhaustivo que se tenga o no de cada uno de ellos.

\section{Nancy avec Badiou: en torno al vacío}

En La desconstrucción del cristianismo, Nancy escribe: 
El sentido se revela y no revela nada, o revela su propia infinidad. Sin embargo, no revelar nada no es una proposición negativa, es más bien la proposición hegeliana en la cual lo que se revela es propiamente que Dios es lo revelable: aquello que es revelado es lo revelable, lo Abierto como tal. (Nancy 2006, p. 55)

Observemos las precauciones terminológicas que toma Nancy, pues son reveladoras, a su vez, de la aproximación y distancia respecto del planteamiento badiousiano del ser.

Primero, la idea fundamental de que aquello que se revela no es nada o, dicho de manera afirmativa, es "nada", o bien su equivalente: "infinidad". Está claro que lo que desea remarcar Nancy antes que nada es que no se trata, en la revelación cristiana, de un objeto determinado (un ente) ni de la simple ausencia de éste: un no-ente. Se trata más bien del acto mismo de revelación que remite a "nada", "infinidad" o "lo Abierto como tal" (todos términos equivalentes en el lenguaje filosófico de Nancy).

Ahora bien, Badiou también se aproxima de algún modo a este motivo por el cual la revelación del ser (o "pensamiento del ser", diría más bien) no atañe a un objeto positivo (propio de una metafísica de la presencia), como tampoco a su simple negación o aproximación diferida (propia de una teología negativa), sino a una multiplicidad pura, inconsistente, sin-uno, aunque rigurosamente circunscrita. Badiou desarrolla este tema de manera exhaustiva en los primeros capítulos de El ser y el acontecimiento, sobre todo en el cuarto capítulo, donde escribe:

desde el momento en que el todo de una situación está bajo la ley de lo uno y de la consistencia, es necesario que, respecto de la inmanencia de una situación, lo múltiple puro, absolutamente impresentable según la cuenta, sea nada. Pero el ser-nada se distingue del no-ser tanto como el "hay" se distingue del ser. (Badiou 1999, p. 69)

El simple e indicativo "hay" remite a dicha multiplicidad inconsistente, pensable por la ontología matemática en términos axiomáticos (axioma del vacío, axioma del infinito, etc.); mientras que el ser-en-situación es siempre múltiple-uno consistente, presentado ónticamente en términos del lenguaje y saber propios de cada situación (ley o estructura). Continúa Badiou:

Así como el estatuto de lo uno se decide entre la tesis (verdadera) "hay uno" y la tesis (falsa) de las ontologías de la presencia "lo uno es", de igual 
manera el estatuto de lo múltiple puro, tomado en la inmanencia de una situación no ontológica, se decide entre la tesis (verdadera) "la inconsistencia es nada" y la tesis estructuralista o legalista (falsa) "la inconsistencia no es". (Badiou 1999, p. 69)

Esto quiere decir que la inconsistencia o la nada se pueden afirmar sea axiomáticamente o sea conceptualmente.

Segundo, el vacío señala así la paradoja del sentido del ser revelado o pensado, su faz afirmativa que lo vincula con la producción genérica infinita de una verdad. ${ }^{1}$ Volvemos a Nancy, quien lo expresa de este modo:

Todo se juega entonces en este punto: pensar la infinidad del sentido, pensar la verdad como infinito del sentido. $O$ aún más, pensar el sentido como una apertura absoluta del sentido y al sentido, pero a un sentido de alguna manera vacío, vacío de todo contenido, de toda figura, de toda determinación. (Nancy 2006, p. 55)

En última instancia: sentido vacío de sentido.

Si bien para Badiou el tratamiento ontológico-matemático del ser en tanto puro vacío está exento de sentido lenguajero (puramente lingüístico), hay que tener en cuenta que Nancy aborda la dimensión del sentido del sentido de una manera muy peculiar que no se identifica con el mero sentido común (de hecho, luego hablará justamente de una "exención de sentido"). Badiou ofrece una precisión conceptual adicional al respecto:

Prefiero decir "vacío" antes que "nada" porque la "nada" es, en todo caso, el nombre del vacío correlativo al efecto global de la estructura (todo es contado), y porque es más preciso indicar que el no-haber-sido-contado es también local, ya que no es contado por uno. "Vacío" indica la falla de lo uno, el no-uno, en un sentido más originario que el no-del-todo. (Badiou 1999, p. 71)

Vacío entonces, en cada caso (local), antes que nada (en forma global).

No obstante, la diferencia fuerte entre Badiou y Nancy surge a partir de que el primero apela a las matemáticas poscantorianas para pensar el vacío en su pura multiplicidad inconsistente, es decir, con el rigor de

${ }^{1}$ Badiou piensa el ser de la verdad como submúltiple genérico de una situación estructurada a partir de los desarrollos matemáticos de Paul Cohen. Véanse los capítulos 31-36 de El ser y el acontecimiento. 
una "teoría consistente de la inconsistencia", mientras que el segundo apela a la "deconstrucción del cristianismo". De este modo se torna inteligible por qué la nada, el vacío, la multiplicidad inconsistente y el infinito actual pueden considerarse, en este nivel de análisis, términos compatibles. Pero, aun así, en lugar de detenerse en una suerte de constatación estática del vacío (la "revelación") o de lo Abierto, la ontología matemática le permite a Badiou tramar múltiples conceptos con dicho vacío (o nombre propio del ser). Podríamos decir que, en lugar de lo abierto o lo infinito, hay múltiples infinitos (infinidad de infinitos) y múltiples aperturas en cada situación óntica singular, localizada históricamente por un acontecimiento y el procedimiento genérico de verdad que le sigue. Así, es posible trabajar el vacío desplazándolo con distintos axiomas y teoremas en la ontología matemática, o bien con nominaciones suplementarias (actos) en los demás procedimientos genéricos de verdad (arte, ciencia, política y amor). Es decir, la revelación (la consumación del nihilismo) no queda fijada bajo la inconsistencia pura del ser (extática), sino en su mismo desplazamiento y torsión entre distintas situaciones singulares y dispositivos de pensamiento para articular otro tipo de consistencia, ya no vinculada exclusivamente con la ley o estructura, como tampoco con el sentido o la representación.

En las preguntas abiertas con las que Nancy finaliza su breve ensayo es donde más se aproxima a la perspectiva filosófica de Badiou; en particular, respecto a la circunscripción del vacío y la producción de una verdad, dice:

Lo abierto (o "lo libre" como lo llamaba también Hölderlin) es, en efecto, esencialmente ambiguo (ésa es la ambigüedad autodestructora o autodesconstructora del cristianismo). En su absolutez, éste se abre sobre sí mismo y no se abre más que sobre sí mismo, infinitamente: así, el cristianismo se hace nihilismo, y se ha comprometido incesantemente con el nihilismo, con la muerte de Dios. Es así que se plantea la siguiente cuestión: ¿qué sería una apertura que no cayese en el abismo de su propio vacío? ¿Qué sería un sentido infinito que, sin embargo, hace del sentido una verdad vacía, que sin embargo posee el peso de una verdad? ¿Cómo trazar nuevamente una apertura delimitada, una figura, que no sea, sin embargo, una captación figurativa del sentido (que no sea Dios) ${ }^{2}{ }^{\text {(Nancy 2006, p. 89) }}$

${ }^{2}$ Giorgio Agamben, un autor que podría ocupar el lugar de mediador entre la perspectiva de Nancy y la de Badiou, también indaga en torno a una nueva figura del sentido, asumiendo el vacío, a través de la noción de "inoperosidad": "hará falta dejar de lado el énfasis sobre el trabajo y sobre la producción e intentar pensar la multitud como figura, si no de la inacción, al menos de un obrar que, en cada 
Como dice Badiou, el acontecimiento es esencialmente la interposición entre el vacío y él mismo (Badiou 1999, p. 231). A partir de esto podríamos responder con Badiou la pregunta formulada por Nancy respecto de una nueva "apertura delimitada": bajo condición de las verdades políticas, científicas, amorosas y artísticas que se interponen, en nominaciones siempre inéditas, entre el vacío ontológico de cada situación singular, nombre propio del ser, y el vacío lógico exento de significación que atañe a cualquier nombre propio. Bajo estas condiciones heterogéneas se con-figura la tarea filosófica de nuestro tiempo, ontología crítica del presente, luego de la muerte de Dios y el nihilismo reinante. Se trata nada más y nada menos que de la praxis de composibilidad; concepto práctico que intentaremos exponer en su complejidad en los apartados siguientes.

2. Lacan avec Badiou: en torno al vacío, el infinito y la diferencia sexual 2.1. Vacío

La diferencia histórica entre Badiou y Lacan se dirime también, según la versión del primero que retoman luego algunos de sus lectores, en función del lugar que ocupa el vacío en cada una de sus teorías. Mientras que el primero piensa el vacío del lado del ser, hasta el punto de constituir su nombre propio, el segundo lo coloca del lado del sujeto: un operador vacío entre significantes:

El lugar donde la filosofía localiza el vacío como condición del pensamiento es el ser en tanto que ser. El lugar donde el psicoanálisis localiza el vacío es el Sujeto, su sujeto, tal como es desvanecido en la separación de los significantes donde procede la metonimia de su ser. (Badiou 2002, p. 264)

Sin embargo, esta simple distinción no basta para pensar la diferencia entre uno y otro (entre Lacan y Badiou, entre sujeto y ser). Hace falta extraer otras consecuencias de ella, remitiéndola al conjunto complejo —esto es, al nudo de conceptos- que se trama entre ambos autores.

acto, realice el propio shabbat y en cada obra sea capaz de exponer la propia inoperosidad y la propia potencia" (Agamben 2007, p. 480). Parte de esa propia potencia es lo que nos lleva a mostrar cómo Agamben, Nancy y Balibar, entre otros, abonan bajo distintos términos u operaciones a una teoría del sujeto que excede toda determinación (i.e., reflexión, identidad, etc.), pues se encuentra en efecto sobredeterminado o anudado, como veremos más adelante. 
En Badiou el nombre propio del ser es en principio el conjunto vacío, el ser-en-tanto-ser en su generalidad antepredicativa es múltiple puro, es decir, múltiple de múltiples, múltiple inconsistente enunciable como veíamos sólo mediante axiomas matemáticos. División incesante, no regulada, de partes en más partes. Pensado de manera aproximada, por fuera del discurso ontológico propiamente dicho, sería la pura dispersión sin límite. Por lo tanto, en Badiou es la operación de sujeto la que fija parcialmente - ya que es una "configuración local finita de un procedimiento de verdad"- - la dispersión absoluta de los términosmúltiples, al forzar a la estructura a contar lo incontado (su verdad) por su infinitud espuria.

En Lacan este proceso se encuentra invertido. Es el sujeto la instancia que encarna la división infinita entre significantes (pues es "aquello que representa el significante para otro significante"), figura evanescente que emerge siempre en eclipse, mientras que la fijación parcial y el índice de la finitud caen del lado de ese ser parcial que es propiamente el objeto $a,{ }^{3}$ es decir, si pretendemos forzar alguna ontología en el discurso del psicoanalista francés, ésta sería del orden del objeto parcial finito, de cuyo estatuto, imposible de aprehender, los movimientos infinitos de subjetivación intentarán dar cuenta de manera siempre fallida.

Es justo en este punto donde ambas posiciones —la de Lacan y la de Badiou-, pese a la inversión, se acercan en una suerte de quiasmo irreductible. Sucede que el sujeto del que habla Badiou no es uno, sino múltiple y cualificado, pues existen al menos cuatro: el artista, el político, el amante, el científico, más un (no-)sujeto, el filósofo, que los piensa conjuntamente. Por lo tanto, si bien un sujeto es una configuración local finita de un procedimiento genérico de verdad, los modos de trazar y pensar las distintas configuraciones singulares pueden ser múltiples infinitos. Por otro lado, si bien el objeto a de Lacan presenta cuatro modos distintos de aparecer (semblantes): voz, mirada, heces y pecho, los modos de circunscribirlo, o sea, los trayectos pulsionales, son infinitos.

Así, vemos cómo se articulan finito e infinito en ambos pensadores y, a la vez, cómo la inversión de los términos no deja de presentar una curiosa compatibilidad. La "diferencia" pasa más bien por los modos

${ }^{3}$ Las únicas innovaciones que, según el propio Lacan, aportó él mismo a la teoría psicoanalítica y cuyas cualidades lo singularizan son tres: ser no especular, ser parcial y ser pulsional. Para aproximarse a la singularidad de este objeto paradójico, clave en la teoría lacaniana, se puede consultar El objeto a de Lacan (Le Gaufey 2013). Dicho objeto no será aquí tema de análisis y desarrollo, pues toca solo tangencialmente nuestra problemática conceptual (quizás como su causa ausente).

Diánoia, vol. LX, no. 74 (mayo de 2015). 
de circulación entre dispositivos de pensamiento que habilitan ambos discursos. Mientras Lacan convoca al arte, la filosofía, la lingüística, las matemáticas, etc., para circunscribir el impasse sexual, Badiou articula de manera equivalente distintos procedimientos genéricos, sin que ninguno de ellos subsuma al otro completamente y constituya una totalidad de sentido; cada problemática singular es, pues, irreductible (real), lo cual no impide el cruce productivo (transferencial) de conceptos.

Por su parte, Marqués Rodilla parte también de la oposición típica señalada entre Badiou y Lacan en torno a la naturaleza problemática del vacío:

Y el conflicto no reside tanto en la amplitud del vacío dado que podría concederse que el vacío del que habla Lacan es un vacío regional que en nada entorpecería un vacío generalizado. El conflicto reside en que el vacío de Lacan es un vacío con bordes, un intervalo perfectamente localizado que se dice como el trayecto que va desde un resto corporal a un objeto topológico. (Marqués Rodilla 2007, p. 330)

Podemos decir que, en Badiou, el vacío no tiene bordes dependiendo del punto de vista que se escoja. Claro que, en el marco de la ontología estricta, el múltiple vacío está suturado a la axiomática conjuntista que impide cualquier normativa del Uno, aunque el "sin borde" no deja de ser "local", como decía Badiou, esto es, circunscrito a la presentación matemática. Por lo tanto, jamás se podría decir que el vacío se encuentra entre dos significantes. Pero esto no es tan simple. En el caso específico de Badiou, tendríamos que exceder el dispositivo ontológico matemático para captar que la vacuidad implicada en él resulta delimitada o acotada de modo complementario por la materialidad de los demás dispositivos de pensamiento, entre los cuales circula la filosofía (que postula la Verdad como operador vacío de composibilidad —y esto remite a esa "apertura delimitada" que mencionaba Nancy-).

Entonces, lo que en verdad tenemos, de uno a otro autor, es un cambio en la amplitud de miras. Dicho de manera simple: en Lacan el sujeto se halla bordeado por los significantes de la lengua materna (la langue) aislados en intensión por el dispositivo analítico, mientras que en Badiou el sujeto se encuentra bordeado por los significantes-demás (suplementarios) producidos en el ámbito extenso de la cultura. Lo que desde el dispositivo psicoanalítico puede captarse del sujeto como corte evanescente entre significantes, en el dispositivo filosófico se capta en cambio entre dispositivos. No tiene sentido analizar un 
concepto aislado del conjunto de dispositivos del que inextricablemente forma parte ni, por consiguiente, de los distintos desplazamientos y (trans)valoraciones que efectúan con ellos Badiou y Lacan. De esto se trata justamente la composibilidad filosófica, la invención conceptual que excede el vacío pues lo circunscribe de múltiples modos.

No obstante, Marqués Rodilla anticipa nuestra tesis sobre el modo de disposición conceptual: "La interconexión entre el acontecimiento, la verdad y el sujeto constituye un nudo borromeo. El nombre del acontecimiento es el resto que ocupa el vacío central del nudo" (Marqués Rodilla 2007, p. 348). Con la salvedad de que, para nosotros, es el sujeto quien ocupa el vacío central del nudo (es el nudo mismo), mientras que el nombre del acontecimiento o intervención es uno más de sus componentes. Retomaremos estos conceptos en el último apartado.

\subsection{Diferencia sexual: el infinito actual y el Dos}

Resulta esclarecedor además seguir la lectura que Badiou hace en su texto "Sujeto e infinito" (2002) de las fórmulas lacanianas de la sexuación, pues se encuentran allí aciertos y desaciertos que enriquecen la comprensión de la compleja posición teórica de ambos autores y de su recurso diferencial a las matemáticas, puntos que nos permitirán aproximarnos mejor a los conceptos adelantados.

Lo primero que llama la atención es que la lectura que realiza Badiou de las fórmulas lacanianas comienza con la expresión "no-toda $x \Phi(x)$ ", del lado femenino, tratando de mostrar así que, para Lacan, al contrario de lo que sucede con la lógica clásica aristotélica, esto no conduce a la afirmación necesaria de una existencia particular: entonces " $\exists x$ no- $\Phi(x)$ ". Pero Badiou no se percata que Lacan procede a la inversa, y construye la universal negativa con el cuantificador existencial $(\exists)$ : "- $\exists x-\Phi(x)$ ", y de ahí se sigue la particular negativa que lo complementa de manera discordante: " $-\forall x \Phi(x)$ ". De este modo, el "no-toda" de la posición femenina resultará afirmado de manera contingente a partir de una excepción inmanente al orden simbólico $\Phi(x)$, pues no hay excepción a priori, ni, por ende, exterioridad alguna. Por eso Lacan menciona la lógica intuicionista, cuya exigencia es que toda existencia debe ser construida, caso por caso, justamente para existir. En su caso, dicha existencia (la del goce femenino infinito) queda indeterminada, o bien sujeta a la verificación de una por una.

Por eso Badiou no es justo con Lacan al decir que éste sigue siendo precantoriano en su uso del infinito actual. Veamos la cita de Lacan en la que se centra Badiou [las cursivas son nuestras]: "La teoría de los 
conjuntos [...] desemboca en lo que ella llama lo no-enumerable [...] pero que al traducirlo a mi vocabulario yo llamo, no lo no-enumerable, objeto que no vacilaría en calificar de mítico, sino la imposibilidad de enumerar" (Lacan en Badiou 2002, p. 281). Dice Badiou al respecto:

Lo vemos: en el caso del continuo, lo infinito actual es decididamente considerado por Lacan como un objeto imaginario. Él se atiene a la función de lo imposible, en sí misma remitida a las operaciones, la enumeración. Aquí se confirma que no hay para Lacan concepto de lo infinito más que en términos de inaccesibilidad operatoria. (Badiou 2002, p. 281)

Resulta curioso que Badiou no se percate de que, justamente, Lacan no se refiere a la existencia del infinito actual (aleph 0), sino al problema del continuo, es decir, a lo que el mismo Badiou denomina el impasse ontológico del ser: la imposibilidad de deducir la cantidad o magnitud del conjunto de partes del infinito actual y no la existencia misma de éste. Dicha imposibilidad o impasse del ser es lo que, como tal, deriva en las distintas orientaciones de pensamiento, incluida la psicoanalítica, según Badiou. Por otra parte, sería pertinente aclarar que Lacan nunca queda sujeto a esa posición mistificadora de un inaccesible. De hecho, si recordamos la definición del matema del significante de la falta del Otro, $\mathrm{S}(\mathbb{A})$, Lacan afirma allí que dicho significante era impronunciable como tal pero no así su operación, que era justamente lo que permitía circunscribirlo cada vez que se producía un nombre propio (Lacan 2003, p. 799). Lacan siempre ha resaltado la dimensión operatoria del acto, incluso la decisión axiomática, en lugar de quedarse en una contemplación mística o mistificante de la cosa imposible.

Esta última es también la vía que adopta Badiou al mostrar que, en tanto el primer cardinal transfinito (aleph 0) es inalcanzable desde las combinaciones operatorias del conjunto de números enteros, entonces su existencia debe decidirse axiomáticamente (el axioma del infinito en el sistema Zermelo-Fraenkel). Badiou lo expresa de manera ejemplar:

Una mujer tendría, como condición de su goce, que decidir lo inaccesible en cuanto a su existencia. Esta dimensión axiomática del goce segundo no contradice en absoluto su carácter indecidible. Ciertamente el axioma enuncia algo, pero la decisión de ese enunciado, el gesto por el cual es inscrito, no son en absoluto enunciados en el axioma mismo. El axioma no dice su dimensión axiomática, y por lo tanto la decisión que él es permanece tácita. (Badiou 2002, p. 283) 
La anterior es otra forma de decir que "No hay metalenguaje" o "No hay relación sexual"; por ende, cada vez hay que decidir, en la inmanencia, sobre el exceso del real en juego.

Badiou se burla un poco de Lacan cuando éste homologa, en la inaccesibilidad, al Dos con el aleph 0; sin embargo, hay algo que se le vuelve a escapar en su lectura. Nuestra hipótesis es que Lacan no se refiere al Dos como número entero (ya que es obvio que uno más uno nos da dos, que no es desde luego inaccesible), sino al concepto de Dos y a cómo se arribó históricamente a él. Ensayemos una aproximación propia de esta problemática.

Suponemos que al principio — de cualquier elaboración simbólicasólo había dos opciones, dos lugares: tener/no tener; presencia/ausencia; positivo/negativo; blanco/negro; 0/1, entonces el juego o la partida inicial es bien simple y repetitivo/a. Suponemos, además, una serie de jugadas: $0 / 1,0 / 1,0 / 1,1 / 0,1 / 0,0 / 1$. Que haya dos posiciones no quiere decir que se sepa qué es el Dos, pues se trata en este caso de una u otra posición consideradas alternativa y excluyentemente. Para captar el Dos, en simultaneidad, es necesario efectuar en cambio un movimiento diacrónico, retroactivo y complementario: volver sobre la jugada anterior y contarla a ella misma; en lugar de 0/1 [...] 0/1 saltar la barra hacia atrás y contar la operación de cuenta anterior como algo distinto de la simple operación sí o no (uno u otro), de cada cuenta, un sí/no más bien (con barra incluida). Puede resultar paradójico que sólo por hacer uno (de $0 / 1)$ se haga dos $([(0 / 1),(0 / 1)])$, pero es que antes eran dos posiciones aisladas (una $u$ otra) y recién al volver hacia atrás y contarlas en conjunto, aunque sean heterogéneas, podemos dar con la diferencia en la repetición. ${ }^{4}$

${ }^{4}$ Esto también se puede expresar en un lenguaje hegeliano, como hace Luciana Cadahia al distinguir entre el infinito espurio y el verdadero:

Podríamos decir que el infinito malo es un círculo vicioso que descansa en la mera enunciación de una contradicción contenida en lo finito. Es decir, algo deviene otro, pero como lo otro también es algo, entonces éste deviene por consiguiente otro (y así hasta el infinito). Lo infinito se reduce a una mera negación de lo finito, dado que se parte de la afirmación de que lo finito es tan algo como su otro, por lo que el infinito progresa como el mero intercambio (una cosa en lugar de otra) de estas determinaciones que conducen (infinitamente) la una a la otra. La verdadera infinitud, nos dice Hegel, sucede cuando algo deviene otro y lo otro deviene lo otro de lo otro mismo. Y a diferencia del infinito malo, aquello a lo que se pasa es enteramente lo mismo que lo que pasa (ambos no tienen otra determinación que una sola y la misma, a saber, la de ser otro). Lo infinito no es contradicción, sino la vuelta a sí del 
Ésta es la radicalidad histórico-mítica del Dos a la que suponemos que, por otros recorridos conceptuales, aludía Lacan, y que se repite en el transfinito cantoriano: ¿cómo pasar de la repetición infinita espuria de los enteros naturales al conjunto transfinito que, al contarlos, marca una diferencia radical y que además, como dice Lacan en el seminario ...Ou pire, se vuelve a producir en torno al problema del continuo y los demás alephs?, es decir que se está moviendo, en su radical conceptualización del goce femenino, en el terreno de los transfinitos cantorianos, sin tenerlos de ningún modo como una mera ficción inaccesible (en todo caso se trataría de una fixión operatoria).

Luego de esta breve confrontación entre Badiou y Lacan en torno a la diferencia (Dos) y al infinito que ella implica (no-todo y no espurio), vamos a sumergirnos en nuestra propia concepción del concepto que, no obstante, es deudora de - pues se apoya en- los planteos anteriores de estos autores y en la mutua imbricación de sus dispositivos de pensamiento.

\section{Estructura y tiempo del concepto: composibilidad entre filosofía y psicoanálisis}

Hay una dificultad central en el planteo badiouano del acontecimiento. Es conocida, pues el mismo Badiou la menciona en el prólogo a la edición en español de El ser y el acontecimiento:

devenir, el llegar a ser algo para sí. Solamente pasando a lo otro algo viene a coincidir consigo mismo y esta referencia a sí mismo en el pasar y en lo otro es la verdadera infinitud. (Cadahia 2015, p. 29)

Y que estas formalizaciones conceptuales en torno al infinito tengan que ver con la sexualidad, más específicamente con la diferencia sexual tal como la encuentra el psicoanálisis, es lo que no se suele entender:

Es decir, aunque la producción de sentido de lo que es ser un "hombre" o una "mujer" es ciertamente simbólica —y masiva - no se constituye para producir diferencia sexual como diferencia significante. En otras palabras, la diferencia sexual es un tipo de diferencia de la diferencia; no sigue la lógica diferencial. Como lo puntualiza Mladen Dolar más sucintamente, la diferencia sexual posa el problema del dos precisamente porque no se puede reducir a la oposición binaria o basarla en términos del binario numérico dos. No es una diferencia significante, tal que defina los elementos de la estructura. (Zupančič 2013, p. 61) 
La doctrina del acontecimiento está marcada por una dificultad interna, enunciada de manera práctica en su misma exposición: si el acontecimiento subsiste sólo porque ha sido objeto de una nominación ¿no hay en realidad dos acontecimientos (el múltiple supernumerario, por un lado, y su nominación, por otro)? [...] Para superar esta dificultad, es necesario complicar un poco el concepto de acontecimiento, dotándolo de una lógica (el acontecimiento es desprendimiento inmediato de una primera consecuencia, tiene una estructura implicativa) y no sólo de una ontológica (el acontecimiento es un múltiple infundado). A su vez esa lógica esclarecerá la potencia propiamente temporal del acontecimiento, la capacidad de engendrar un tiempo propio. (Badiou 1999, p. 7)

La paradoja o circularidad que puede hallarse en Badiou entre los conceptos de acontecimiento y de intervención no puede resolverse definitivamente, pues en la misma producción conceptual está implícita una dimensión temporal que, esencialmente abierta, nos conduce a escindir el círculo y, a la vez, a anudarlo con otros círculos (conceptos). Así, es posible a lo sumo asumir su complejización y desplazamiento a partir de nuevos conceptos (círculos) y dispositivos, tal como lo hace Badiou en Lógicas de los mundos al incorporar, por ejemplo, la teoría de las categorías para pensar los dos tiempos de producción del acontecimiento (autopertenencia del sitio acontecimental y consecuencias postacontecimentales). Por nuestra parte, escogemos insistir sobre el anudamiento de las categorías anteriores a la aparición de Lógicas de los mundos; nos referimos al complejo temático integrado por los conceptos de acontecimiento, intervención, sujeto, operador de conexión y verdad. ${ }^{5}$

Vamos a tratar de redescribir conceptualmente la lógica paradojal del acontecimiento y su nominación. Primero, el acontecimiento es un múltiple cuya pertenencia a la situación resulta indecidible, es decir, que no es inmanente a ella ni tampoco trascendente. Por lo tanto, para existir requiere de una decisión infundada que afirme su pertenencia a la situación. De esto se trata la nominación suplementaria — pues no corresponde a las correlaciones significantes establecidas- $\mathrm{o}$ intervención. Segundo, es en virtud de dicha intervención efectiva que, retroactivamente, se constituye un sujeto, y no al revés. Ahora bien, ¿quién interviene si no hay sujeto hasta el momento de la misma intervención? Además, sumado al problema anterior, el acontecimiento no

${ }^{5}$ Estas cuestiones conceptuales, bastante complejas, las desarrolla el propio Badiou entre los capítulos 16 y 23 de El ser y el acontecimiento. Aquí no buscaremos definir explícitamente cada uno de los términos teóricos implicados, pues nos llevaría a repetir los capítulos aludidos, sino ensayar una hipótesis sobre su dialéctica, su modo de articulación conjunta. 
habrá existido —no habrá tenido lugar- si no es por la intervención que lo nombra y lo pone a circular en situación; pero, a su vez, esta misma intervención tampoco es posible si no hay acontecimiento (no es voluntarista). Parece entonces que ambos conceptos, acontecimiento e intervención, junto al concepto de sujeto, se presuponen mutuamente. $\mathrm{Y}$ así pues, sucede.

Acto seguido hay que explicar cómo se da esta implicación mutua: el nudo temporal del concepto. Esta formulación ya no pertenece a Badiou, sino que constituye nuestra propia hipótesis de lectura respecto a la composibilidad conceptual entre filosofía y psicoanálisis, apelando para ello a la estructura topológica del nudo borromeo. Como mencionamos antes, según Badiou, la nominación del acontecimiento se interpone entre el vacío intrasituacional y el acaecer contingente del mismo acontecimiento (Badiou 1999, p. 228). Podríamos forzar pues, en una suerte de aproximación metaontológica, que el acontecimiento no es sino un efecto de interposición (entre el vacío y su nombre). Tenemos por lo tanto tres términos coimplicados allí: i) nombre, ii) vacío, iii) acontecimiento. Tal es la primera articulación conceptual triple $\mathrm{y}$, por ende, "ontológica".

Después, al tratar de especificar a partir de qué se autoriza la intervención subjetiva (para no caer en el izquierdismo especulativo y voluntarista del comienzo absoluto), nos encontramos con una segunda estructuración triple que, retroactivamente, se descubre que antecede a la que suponíamos primera: "Para evitar la curiosa remisión en espejo del acontecimiento y la intervención —del hecho y la interpretación-, es necesario atribuir la posibilidad de intervención a las consecuencias de otro acontecimiento [...] La intervención presenta un acontecimiento para el advenimiento de otro. Es un entre-dos acontecimental" (Badiou 1999, p. 234); la intervención-nominación opera en nombre propio, ${ }^{6}$ el cual hace así las veces de mediador entre el mismo acontecimiento

${ }^{6}$ Así lo expresa Badiou:

La subjetivación, configuración singular de una regla, subsume al Dos que ella es en la ausencia de significación de un nombre propio. San Pablo para la Iglesia, Lenin para el Partido, Cantor para la ontología, Schoenberg para la música, pero también Simon, Bernard o Claire, si ellos declaran un amor: son designaciones a través de lo uno de un nombre propio, de la escisión subjetivadora entre el nombre de un acontecimiento (muerte de Dios, revolución, múltiples infinitos, destrucción del sistema tonal, encuentro) y la puesta en marcha de un procedimiento genérico (Iglesia cristiana, bolchevismo, teoría de los conjuntos, serialismo, amor singular). (Badiou 1999, p. 433) 
y otros. Entonces, tenemos: i) nombre del acontecimiento, ii) nombre propio, iii) algunos otros acontecimientos (nombres propios). Es decir que se descubren, en el mismo acto de nominación, una serie de acontecimientos dispares, heterogéneos, que autorizan la intervención histórica y constituyen una suerte de "tradición subterránea del materialismo del encuentro", podríamos decir siguiendo a Althusser (2002). Ésta es la segunda articulación conceptual triple y, por ende, "histórica".

Pero dicha temporalidad se despliega no sólo hacia atrás, abierta así hacia el pasado reactualizado de una serie virtualmente infinita de nombres propios, sino también hacia adelante, prospectivamente, en los procesos de indagación postacontecimentales ligados a la fidelidad:

Es necesario retener y fijar conceptualmente que una fidelidad queda definida, de manera conjunta, por: una situación - aquella en la que se encadenan los efectos de la intervención, según la ley de la cuenta-, un múltiple particular - el acontecimiento tal como fue nombrado y puesto en circulación-y una regla de conexión que permite evaluar la dependencia de cualquier múltiple existente respecto del acontecimiento, de acuerdo a cómo la intervención decidió su partencia a la situación. (Badiou 1999, p. 261)

Aquí encontramos la tercera articulación triádica, que podríamos llamar "práctica" o "institucional": i) nombre del acontecimiento, ii) operador de conexión fiel, iii) múltiples indagados al azar. En cada nueva indagación se reactualiza la serie de nombres propios en torno a los cuales se teje el orden acontecimental y su historicidad intrínseca; tradición materialista subterránea de pensamiento que no se deja identificar por rasgos imaginarios sino por intervenciones efectivas.

Así, en cada intervención y en cada indagación postacontecimental se reactualiza la doble apertura (doble bucle) temporal: retrospectiva y prospectiva. Pero, además, se efectúa una sutura local (actual) por intermedio del anudamiento entrecruzado de estos tres términos de tres. Una estructura nodal compleja trama estos conceptos abiertos a la temporalidad. Se podría pensar estáticamente que son tres estructuras espaciales de tres términos cada una o quizá una sola que se repite con variaciones, pero dicha interpretación (o cuenta errónea) pasaría por alto que es justamente la dimensión temporal la que abre el espacio cerrado de tres términos, tanto hacia atrás como hacia adelante, por lo que habría que contarla más bien como la cuarta dimensión suplementaria de la estructura conceptual topológica. Podríamos decir que cada intervención, cada nominación, cada conexión fiel, reactualiza en parte el orden acontecimental que toda situación estructuralmente ignora. 
Incluso se podría suponer antes una estructura triádica o tri-vial propiamente ontológica: i) estructura presentacional, ii) metaestructura representacional, y iii) vacío impresentable, pues en los comentarios ontológicos Badiou anticipa de algún modo cómo se juega lo acontecimental histórico en este plano que en principio lo excluye. Así, el acontecimiento es la dislocación misma entre estructura y metaestructura, que aparece en la segunda escena triádica: i) sitio del acontecimiento o vacío intrasituacional óntico, ii) acontecimiento, y iii) intervención que lo nombra. El acontecimiento es ese "mediador evanescente" que se constituye suplementariamente a partir de la intervención, que no es voluntarista pues se autoriza a partir de otros acontecimientos pasados y porvenir (recurrencia acontecimental y confianza, es "entre-dos"). Se abre así la tercera escena, que da inicio a lo propiamente postacontecimental de un procedimiento genérico de verdad: i) nombre del acontecimiento, ii) operador de conexión fiel (el sujeto es el Dos, entre ellos) y iii) múltiple indagado. De este modo, se aprecia en estas modulaciones conceptuales cómo la inestabilidad de una estructura triádica es anticipada por un término evanescente y suplementario que aparece en la próxima escena e introduce la apertura temporal, más uno que se repite y otro novedoso.

Estas articulaciones — tetrádicas espacio-temporales- sobre la lógica del acontecimiento nos servirán, a su vez, para repensar la dinámica de un análisis. Del mismo modo, podemos situar en un primer momento al analista cara a cara con el analizante y entender así cómo resulta incidida dicha dualidad imaginaria, propia de las entrevistas preliminares, por la entrada efectiva en análisis y el emerger - en cierta forma acontecimental— del sujeto dividido por el significante que requiere la operación analítica. Quizá lo que cumpla la función del tercero relativo sea, prima facie, el mismo dispositivo y su efectividad simbólica: la teoría psicoanalítica, los nombres propios de Freud y Lacan, el supervisor, la escuela, la institución, etcétera.

Ahora bien, ¿cómo suspender el saber ante cada caso singular, tal como recomendaba Freud? Pensemos en términos badiousianos. Cada individuo, aún no devenido sujeto dividido, es múltiple de múltiples, infinito actual, en su estricta constitución ontológica. El asunto es que, en situación normal, todo múltiple es contado por uno como elemento y a la vez como parte. Es presentado y representado. Elemento de estructura (simbólica) y parte de metaestructura (imaginaria). Dicha conjugación, objetivante por naturaleza, es la que oblitera la división constitutiva (en términos lacanianos) o la pura multiplicidad (en términos badiousianos). Por lo tanto, se tratará de habilitar el acontecimiento de 
la división subjetiva (dislocación entre estructura y metaestructura, entre simbólico e imaginario) y el devenir múltiple genérico del ser en situación: la nominación de lo incontado en un procedimiento de verdad.

Aquí es donde la triple estructuración triádica mencionada antes nos puede orientar. Podemos imaginar que no es operativo que la estructura triádica quede suspendida sólo en el primer tiempo, por ejemplo entre el analista, el analizante y Freud, como supuesto modelo de intervención terapéutica que se sigue (o la teoría psicoanalítica, como saber ya sabido, clasificatorio, tipológico, etc.); no es operativo porque mantiene la estructura de manera estática (estatal y representativa), sin incluir las otras dimensiones de la apertura temporal aludidas antes y, por ello, las intervenciones seguramente serán rígidas, esquemáticas, estereotipadas y no tendrán efecto sobre lo real (o no, al menos, un efecto acontecimental verdadero, quizá sí un efecto terapéutico pasajero). En cambio, si el analista ha pasado él mismo por un proceso sostenido de división subjetiva (su propio análisis) y a partir de la nominación de dicho acontecimiento ha conectado su ser-múltiple-genérico con otros nombres propios (e.g., Freud-Lacan), entonces resultará habilitado para indagar, junto con el singular múltiple-individuo, su propia capacidad in-sabida para soportar la división acontecimental; lo que constituirá un sujeto de la verdad analítica en proceso.

Así pues, las estructuras geométricas rígidas de triangulación (más bien epistemológica) — teoría (modelo), caso (analizante), aplicación (analista) - se ven profundamente afectadas y descentradas por la dimensión temporal retro y prospectiva que flexibiliza la dinámica estructural y la topologiza (u ontologiza). Hay un pasaje de una geometría de sólidos (triangular) a una topología flexible de cuerdas y anudamientos (toros), donde el orden no es jerárquico y rígido, sino alternado y variable: Freud y Lacan son sólo otros tantos nombres acontecimentales - trazas supernumerarias- de lo real evanescente, entre lo que ha sido y lo porvenir. El momento de la división subjetiva es acontecimental, en efecto, si la falla de la estructura (des-objetivación) es nombrada, no sólo en alusión al vacío intrasituacional (que recubre el fantasma) sino en el anudamiento mismo que circunscribe dicho vacío con otros nombres acontecimentales, producidos y por producir (tradición subterránea cuya emergencia sucede siempre en acto).

Con el nudo borromeo podemos trazar el recorrido imbricado de los tres registros: i) la dualidad imaginaria (yo/otro) resulta escindida por la terceridad relativa que introduce lo simbólico (un significante para otro significante); ii) a su vez este eterno diferir significante en pos de la significación resulta suspendido por la insensatez apremiante de lo real 
(el afecto), y iii) finalmente éste es suplementado por un nuevo imaginario que, a diferencia del primero, anuda tanto lo simbólico como lo real y, por lo tanto, es heterogéneo.

Finalmente hemos podido apreciar cómo la cuestión primaria de la vacuidad, del infinito, de la apertura, en un recorrido zigzagueante por otros decursos conceptuales y en parte formales, trama la complejidad misma que la elabora, sin contentarse con el mero abismo sin fondo. Esto señala nuestra apuesta por la composibilidad nodal del concepto en la consumación del nihilismo contemporáneo.

\section{BIBLIOGRAFÍA}

Agamben, G., 2007, La potencia del pensamiento. Ensayos y conferencias, Adriana Hidalgo, Buenos Aires.

Althusser, L., 2002, Para un materialismo aleatorio, trad. P. Fernández Lira, L. Alegre Zahonero y G. González Diéguez, Arena, Madrid.

Badiou, A., 2008, Lógicas de los mundos: el ser y el acontecimiento 2, trad. M. del C. Rodríguez, Manantial, Buenos Aires.

— 2002 , Condiciones, trad. E.L. Molina y Vedia, Siglo XXI, Buenos Aires.

-, 1999, El ser y el acontecimiento, trad. R.J. Cerdeiras, A.A. Cerletti y N. Prados, Manantial, Buenos Aires.

Cadahia, M.L., 2015, "El pensamiento especulativo en clave contemporánea: Hegel y la filosofía de la diferencia", Daimon. Revista Internacional de Filosofía, vol. 64, pp. 23-34.

Lacan, J., 2012, El Seminario de James Lacan. Libro 19: ... O peor. 1971-1972, trad. G. Arenas, Paidós, Buenos Aires.

- 2003, Escritos 1 y 2, trad. T. Segovia, Siglo XXI, Buenos Aires.

Le Gaufey, G., 2013, El objeto a de Lacan, trad. N. Pasternac, El Cuenco de Plata, Buenos Aires.

Marqués Rodilla, C., 2007, “Sobre el sujeto: ¿Deleuze versus Badiou?”, Éndoxa: Series Filosóficas, no. 22, pp. 325-356.

Nancy, J.-L., 2006, La desconstrucción del cristianismo, trad. A. Madrid Zan, La Cebra, Buenos Aires.

Zupančič, A., 2013, La sexualidad dentro de los límites de la razón, trad. P. Pérez Wilson, Palinodia, Santiago de Chile.

Recibido el 30 de enero de 2014; aceptado el 2 de marzo de 2015. 